\title{
NON-LEFT-COMPLETE DERIVED CATEGORIES
}

\author{
AMNON NeEMAN
}

Abstract. We give some examples of abelian categories $\mathcal{A}$ for which the derived category $\mathbf{D}(\mathcal{A})$ is not left-complete. Perhaps the most natural of these is where $\mathcal{A}$ is the category of representations of the additive group $\mathbb{G}_{a}$ over a field $k$ of characteristic $p>0$.

\section{Contents}

0. Assumed background

1. The counterexample

\section{Assumed background}

In this article, we assume the reader is familiar with derived categories and with $t$-structures on them. See Verdier [5] for the theory of derived categories, and Beilinson et al. [1, Chapter 1] for an introduction to $t$-structures.

\section{The counterexample}

Suppose $\mathcal{A}$ is an abelian category and $\mathbf{D}(\mathcal{A})$ is its derived category. For any object $x \in$ $\mathbf{D}(\mathcal{A})$, we write $x^{\geq n}$ for the truncation of $x$ with respect to the standard $t$-structure. We have canonical maps $x^{\geq n} \longrightarrow x^{\geq n+1}$, and a (non-canonical) map

$$
\varphi_{x}: x \longrightarrow \underset{4}{\text { Holim }} x^{\geq n} .
$$

The category $\mathbf{D}(\mathcal{A})$ is said to be left-complete if, for every object $x \in \mathbf{D}(\mathcal{A})$, any map $\varphi_{x}$ as above is an isomorphism. Even though the map $\varphi_{x}$ is not canonical, it can be shown that, for given $x$, if one $\varphi_{x}$ is an isomorphism then they all are.

The reader can find much more about left-complete categories in Lurie [3, Section 7] or [4, Subsection 1.2.1, more precisely starting from Proposition 1.2.1.17]. See also Drinfeld and Gaitsgory [2].

In this note we will see how to produce many $\mathcal{A}$ for which $\mathbf{D}(\mathcal{A})$ is not left-complete. Our counterexamples will be of a very special form, which allows us to easily compute the homotopy inverse limit $\operatorname{Holim} x^{\geq n}$. Let us now sketch what we will do.

Received by the editors April 15, 2011.

2000 Mathematics Subject Classification. Primary 18E30, secondary 18G55.

Key words and phrases. Derived categories, $t$-structures, homotopy limits. 
We will suppose that the abelian category $\mathcal{A}$ satisfies the axiom [AB4], that is, coproducts are exact; this makes it easy to compute coproducts in the derived category $\mathbf{D}(\mathcal{A})$, just form the coproduct as complexes. Suppose $A$ is an object in our [AB4] abelian category $\mathcal{A}$, and let

$$
x=\coprod_{i=0}^{\infty} A[i] .
$$

It is clear that, for $n>0$, we have

$$
x^{\geq-n}=\coprod_{i=0}^{n} A[i]=\prod_{i=0}^{n} A[i],
$$

where the last equality is because finite coproducts agree with finite products. Now the homotopy inverse limit of the products is a genuine inverse limit, and we have

$$
\text { Holim } x^{\geq n}=\prod_{i=0}^{\infty} A[i] .
$$

Thus, our problem reduces to deciding whether the map

$$
\coprod_{i=0}^{\infty} A[i] \stackrel{\varphi}{\longrightarrow} \prod_{i=0}^{\infty} A[i]
$$

is an isomorphism. Note that in this case the map is canonical; our homotopy inverse limit happens to be a genuine inverse limit, removing the arbitrariness. The left-hand side is easy to work with; its cohomology is $A$ in each degree $n \leq 0$. What we will show is how to produce examples where the right-hand side has a lot of more cohomology. More precisely, we have

$$
\prod_{i=0}^{\infty} A[i]=A[0] \oplus\left(\prod_{i=1}^{\infty} A[i]\right)
$$

and the expectation would be for the second term to have a vanishing $H^{0}$; what we will show is how to produce non-zero classes in

$$
H^{0}\left(\prod_{i=1}^{\infty} A[i]\right) .
$$

It is time to disclose what will be our choice for the category $\mathcal{A}$ and for the object $A \in \mathcal{A}$.

Construction 1.1. Let $k$ be a field, let $R_{1}$ be a finitely generated $k$ algebra, and let $\mathfrak{m}$ be a $k$-point of $\operatorname{Spec}\left(R_{1}\right)$. In other words, $\mathfrak{m} \subset R$ is a maximal ideal with $R_{1} / \mathfrak{m} \cong k$. We make a string of definitions:

(i) $R_{n}=\otimes_{i=1}^{n} R_{1}$, where the tensor is over the field $k$.

(ii) The inclusion $R_{n} \longrightarrow R_{n+1}$ is the inclusion of the tensor product of the first $n$ terms.

(iii) $R=\underset{\operatorname{colim}}{\longrightarrow} R_{n}$.

(iv) The map $\Phi_{i}: R_{1} \longrightarrow R$ is the inclusion of the $i$ th factor.

(v) The category $\mathcal{A}$ will be the category of all those $R$-modules, on which $\Phi_{i}(\mathfrak{m})$ acts trivially for all but finitely many $i$.

The object $A \in \mathcal{A}$ will be the colimit over $n$ of the $R_{n}-$ modules $k=\otimes_{i=1}^{n}\left[R_{1} / \mathfrak{m}\right]$. 
The main result is

Theorem 1.1. Assume that $k=R_{1} / \mathfrak{m}$ is not projective over the localization $\left(R_{1}\right)_{\mathfrak{m}}$ of the ring $R_{1}$ at the maximal ideal $\mathfrak{m}$. With the category $\mathcal{A}$ and the object $A \in \mathcal{A}$ as in Construction 1.1, there is a non-zero element in

$$
H^{0}\left(\prod_{i=1}^{\infty} A\right)
$$

Remark 1.1. The case where $R_{1}=k[x] /\left(x^{p}\right)$ is of particular interest. If the field $k$ is of characteristic $p$ then the category $\mathcal{A}$ happens to be the category of representations of the additive group $\mathbb{G}_{a}$, and we learn that its derived category is not left-complete.

Remark 1.2. We trivially have

$$
\prod_{i=1}^{\infty} A[i]=\left(\prod_{i=1}^{n} A[i]\right) \oplus\left(\prod_{i=n+1}^{\infty} A[i]\right)
$$

and hence

$$
H^{0}\left(\prod_{i=1}^{\infty} A[i]\right)=H^{0}\left(\prod_{i=1}^{n} A[i]\right) \oplus H^{0}\left(\prod_{i=n+1}^{\infty} A[i]\right) .
$$

On the other hand, with the finite product we have no problem computing

$$
H^{0}\left(\prod_{i=1}^{n} A[i]\right)=H^{0}\left(\coprod_{i=1}^{n} A[i]\right)=0,
$$

and Theorem 1.1 now allows us to deduce that

$$
H^{0}\left(\prod_{i=n+1}^{\infty} A[i]\right) \neq 0 \text {. }
$$

Translating, we have

$$
H^{n}\left(\prod_{i=1}^{\infty} A[i]\right) \neq 0
$$

for all $n \geq 0$. The complexes $A[i], i>0$ all belong to $\mathbf{D}(\mathcal{A})^{<0}$, but the product $\prod_{i=1}^{\infty} A[i]$ is not bounded above.

\section{The proof}

We begin with a little lemma.

Lemma 2.1. Let $k$ be a field, and let $R$ and $S$ be finitely generated $k$-algebras. Suppose further that we are given $k$-points of $\operatorname{Spec}(R)$ and $\operatorname{Spec}(S)$; that is $\mathfrak{m} \subset R$ and $\mathfrak{n} \subset S$ are maximal ideals, with

$$
R / \mathfrak{m} \cong k \cong S / \mathfrak{n}
$$

Let $E$ be an injective envelope of $k=R / \mathfrak{m}$ over the ring $R$, and $F$ an injective envelope of $k=S / \mathfrak{n}$ over the ring $S$. Then $E \otimes_{k} F$ is an injective envelope of $k$ over the ring $R \otimes_{k} S$. 
Proof. We will first prove the case where $R$ and $S$ are polynomial rings.

Let $R^{\prime}=k\left[x_{1}, x_{2}, \ldots, x_{m}\right]$ be a polynomial ring, and let $\mathfrak{m}$ be the maximal ideal generated by $\left\{x_{1}, x_{2}, \ldots, x_{m}\right\}$. Then we know the injective envelope $E^{\prime}$ of $k=R^{\prime} / \mathfrak{m}$ explicitly: it is the quotient of $S=k\left[x_{1}, x_{1}^{-1}, x_{2}, x_{2}^{-1}, \ldots, x_{m}, x_{m}^{-1}\right]$ by the $R^{\prime}$-submodule generated by all monomials $x_{1}^{i_{1}} x_{2}^{i_{2}} \cdots x_{m}^{i_{m}}$ with at least one of the $i_{j}>0$. As a $k$-vector space $E^{\prime}=k\left[x_{1}^{-1}, x_{2}^{-1}, \ldots, x_{m}^{-1}\right]$, and the $R^{\prime}$-module structure is obvious when we declare $x_{1}^{i_{1}} x_{2}^{i_{2}} \cdots x_{m}^{i_{m}}=0$ if some $i_{j}>0$. If $S^{\prime}=k\left[y_{1}, y_{2}, \ldots, y_{n}\right]$ and $\mathfrak{n} \subset S^{\prime}$ is the ideal generated by $\left\{y_{1}, y_{2}, \ldots, y_{n}\right\}$, then the fact that

$$
E^{\prime} \otimes_{k} F^{\prime}=k\left[x_{1}^{-1}, x_{2}^{-1}, \ldots, x_{m}^{-1}\right] \otimes_{k} k\left[y_{1}^{-1}, y_{2}^{-1}, \ldots, y_{n}^{-1}\right]
$$

is the injective hull of $k$ over $R^{\prime} \otimes S^{\prime}$ is by inspection.

Now for the general case: assume $R=R^{\prime} / I$ and $S=S^{\prime} / J$ where $R^{\prime}$ and $S^{\prime}$ are polynomial rings, and $I \subset R^{\prime}$ and $J \subset S^{\prime}$ are ideals contained in the $\mathfrak{m}$ and $\mathfrak{n}$ above. Then the injective hull $E$ of $k=R / \mathfrak{m}$ over the ring $R$ is the largest $R$-submodule of the $R^{\prime}$-module $E^{\prime}$, that is, the $R^{\prime}$-submodule $E \subset E^{\prime}$ of all elements annihilated by the ideal $I$. The lemma therefore comes down to the fact that the submodule of $E^{\prime} \otimes_{k} F^{\prime}$ annihilated by the ideal $I \otimes_{k} S^{\prime}+R^{\prime} \otimes_{k} J$ is precisely $E \otimes_{k} F$.

Proof of Theorem 1.1. Let $\bar{R}$ be the localization of $R_{1}$ at the maximal ideal $\mathfrak{m}$. We are assuming that $k$ is not projective over $\bar{R}$, that is the projective dimension of $k$ is at least one. Choose and fix a minimal free resolution of $k=\bar{R} / \mathfrak{m} \bar{R}$ as an $\bar{R}$-module. Let us write this resolution as

$$
P_{2} \longrightarrow P_{1} \longrightarrow P_{0} \longrightarrow k \longrightarrow 0 \text {. }
$$

Then the modules $P_{i}$ are all finite and free over the ring $\bar{R}$, the differentials are all matrices over $\bar{R}$, and the minimality guarantees that the entries in these matrices all belong to the ideal $\overline{\mathfrak{m}}=\mathfrak{m} \bar{R} \subset \bar{R}$. Now let $E$ be the $\bar{R}$-injective envelope of the module $k$; applying the functor $\operatorname{Hom}_{\bar{R}}(-, E)$ to the projective resolution above, we produce an injective resolution $I^{*}$ of $k$, which we write as

$$
0 \longrightarrow k \longrightarrow I^{0} \longrightarrow I^{1} \longrightarrow I^{2}
$$

We know that each $I^{j}=\operatorname{Hom}\left(P_{j}, E\right)$ is a finite coproduct of copies of $E$, and that the differentials $I^{j} \longrightarrow I^{j+1}$ are matrices whose entries belong to the ideal $\overline{\mathfrak{m}}$. The fact that the projective dimension of $k$ is at least one tells us that $P_{1} \neq 0$, and therefore $I^{1} \neq 0$. Note that an injective envelope $E$ of $k$ over the localized ring $\bar{R}=\left(R_{1}\right)_{\mathfrak{m}}$ is also an injective envelope of $k$ over the ring $R_{1}$, and hence we have produced an injective resolution of $k$ over $R_{1}$. Next we

(i) Choose a non-zero element $a$ in the image of the map $k \longrightarrow I^{0}$.

(ii) Choose a non-zero element $b \in I^{1}$, with $\mathfrak{m} b=0$.

If we view $k$ as a module over the $\operatorname{ring} R_{n}=\otimes_{i=1}^{n} R_{1}$, then the tensor product $J_{n}^{*}=\otimes_{i=1}^{n} I^{*}$ is certainly a resolution of $k$ as an $R_{n}$ module, and Lemma 2.1 guarantees further that

(iii) Each $J_{n}^{i}$ is injective as a module over $R_{n}$.

(iv) Let the inclusion $J_{n}^{*} \longrightarrow J_{n+1}^{*}$ be the map taking $x \in J_{n}^{*}$ to

$$
x \otimes a \in J_{n}^{*} \otimes I^{0} \subset J_{n}^{*} \otimes I^{*}=J_{n+1}^{*},
$$


where $a \in I^{0}$ is as in (i) above. We define $J^{*}$ to be

$$
J^{*}=\underset{\operatorname{colim}}{\longrightarrow} J_{n}^{*}
$$

then $J^{*}$ is an injective resolution of $k$ in the category $\mathcal{A}$.

To prove the theorem we need to find a non-zero element in $H^{0}\left(\prod_{i>0} k[i]\right)$, and our next observation is that the product in the derived category $\prod_{i>0} k[i]$ is obtained as the the ordinary product of injective resolutions. The complex $J^{*}[i]$ is an injective resolution of $k[i]$, and hence the derived product $\prod_{i>0} k[i]$ is just the usual product $\prod_{i>0} J^{*}[i]$. Now for every $i \geq 1$ let

$$
S_{i}=\left\{i^{2}+1, \ldots, i^{2}+i\right\},
$$

and observe that the sets $S_{i}$ are disjoint. In the injective $R_{i^{2}+i^{-}}$-module

$$
J_{i^{2}+i}^{i}=\coprod_{\sum \ell_{m}=i} I^{\ell_{1}} \otimes I^{\ell_{2}} \otimes \cdots \otimes I^{\ell_{i^{2}+i}}
$$

or more specifically in the summand

$$
\left(I^{0}\right)^{\otimes i^{2}} \otimes\left(I^{1}\right)^{\otimes i}
$$

we take the term

$$
\lambda_{i}=a^{\otimes i^{2}} \otimes b^{\otimes i}
$$

where $a \in I^{0}$ and $b \in I^{1}$ are as in (i) and (ii) above. The embedding $J_{i^{2}+i}^{*} \longrightarrow J^{*}$ of (iv) gives us an element which we will denote $\lambda_{i} \in J^{i}$. The elements $\lambda_{i}$ have the properties

(v) Each $\lambda_{i}$ is a cycle; the differential $J^{i} \longrightarrow J^{i+1}$ kills $\lambda_{i}$.

(vi) $\Phi_{j}(\mathfrak{m}) \lambda_{i}=0$ for all $i$ and $j$.

We are assuming $i>0$, so each $\lambda_{i}$ must be a boundary because $H^{i}\left(J^{*}\right)=0$. But if $\mu_{i} \in J^{i-1}$ maps to $\lambda_{i}$, then there must exist an integer $j \in S_{i}$ so that $\Phi_{j}(\mathfrak{m})$ does not kill $\mu_{i}$. Now form the element

$$
\prod_{i=1}^{\infty} \lambda_{i} \in \prod_{i=1}^{\infty} J^{i}
$$

where the product is in the category of all $R$-modules.

Caution 2.1. The reader is reminded that the category $\mathcal{A}$ is a subcategory of the category of $R$-modules. Both categories have infinite products; the products in the category of $R$-modules are just the usual cartesian products, while the products in $\mathcal{A}$ are subtler. To form the product in $\mathcal{A}$ of a bunch of objects in $\mathcal{A}$, one first forms the usual cartesian product, and then considers inside it the largest object belonging to $\mathcal{A}$, that is the collection of all elements satisfying part (v) of Construction 1.1.

The element $\prod_{i=1}^{\infty} \lambda_{i}$ is a degree 0 cycle in the complex $\prod_{i \geq 1} J^{*}[i]$, and it is annihilated by $\Phi_{j}(\mathfrak{m})$ for all $j$. By Caution 2.1 we have that $\prod_{i=1}^{\infty} \lambda_{i}$ belongs to $\prod_{i=1}^{\infty} J^{i}$ even when the product is understood in $\mathcal{A}$. However, it is not a boundary in $\mathcal{A}$. If we try to express $\prod_{i=1}^{\infty} \lambda_{i}$ as the boundary of

$$
\prod_{i=1}^{\infty} \mu_{i} \in \prod_{i=1}^{\infty} J^{i-1}
$$


then we discover that each $\mu_{i}$ fails to be annihilated by some $\Phi_{j}(\mathfrak{m})$ with $j \in S_{i}$. As the $S_{i}$ are disjoint, this produces infinitely many $\Phi_{j}(\mathfrak{m})$ not annihilating $\prod_{i=1}^{\infty} \mu_{i}$, meaning that it does not belong to $\mathcal{A}$.

\section{Acknowledgments}

The author would like to thank Drinfeld and Gaitsgory for asking the question that led to these counterexamples. The research was partly supported by the Australian Research Council.

\section{References}

[1] A. A. Beilinson, J. Bernstein, and P. Deligne, Analyse et topologie sur les éspaces singuliers, Astérisque, Soc. Math. France, 100, 1982.

[2] V. Drinfeld and D. Gaitsgory, On some finiteness questions for algebraic stacks, arXiv: $1108.5351 \mathrm{v} 1$.

[3] J. Lurie, Derived algebraic geometry I: stable $\infty$-categories, arXiv:math/0608228v5.

[4] — Higher Algebra, prepint (available from http://www.math.harvard.edu/ lurie/).

[5] J.-L. Verdier, Des catégories dérivées des catégories abeliennes, Asterisque, Société Mathématique de France, 239, 1996.

Centre for Mathematics and its Applications, Mathematical Sciences Institute, John Dedman Building, The Australian National University, Canberra, ACt 0200, AUStralia E-mail address: Amnon.Neeman@anu.edu.au 